\title{
Color critical hypergraphs and forbidden configurations
}

\author{
Richard Anstee $^{1 \dagger}$, Balin Fleming $1 \ddagger$ Zoltán Füredi ${ }^{23 \S}$ and Attila Sali ${ }^{3 థ}$ \\ ${ }^{1}$ Mathematics Department The University of British Columbia Vancouver, B.C., Canada V6T 1Z2 \\ ${ }^{2}$ Department of Mathematics University of Illinois at Urbana-Champaign 1409 W. Green Street Urbana, Illinois \\ 61801-2975, USA \\ ${ }^{3}$ Alfréd Rényi Instiute of Mathematics Hungarian Academy of Sciences Budapest, P.O.Box 127 H-1364 Hungary
}

The present paper connects sharpenings of Sauer's bound on forbidden configurations with color critical hypergraphs. We define a matrix to be simple if it is a $(0,1)$-matrix with no repeated columns. Let $F$ be a $k \times l(0,1)$-matrix (the forbidden configuration). Assume $A$ is an $m \times n$ simple matrix which has no submatrix which is a row and column permutation of $F$. We define forb $(m, F)$ as the best possible upper bound on $n$, for such a matrix $A$, which depends on $m$ and $F$. It is known that forb $(m, F)=O\left(m^{k}\right)$ for any $F$, and Sauer's bond states that forb $(m, F)=O\left(m^{k-1}\right)$ fore simple $F$. We give sufficient condition for non-simple $F$ to have the same bound using linear algebra methods to prove a generalization of a result of Lovász on color critical hypergraphs.

Keywords: forbidden configuration, color critical hypergraph, linear algebra method

\section{Introduction}

A $k$-uniform hypergraph $(V, \mathcal{E})$ is 3-color critical if it is not 2-colorable, but for all $E \in \mathcal{E}$ the hypergraph $(V, \mathcal{E} \backslash\{E\})$ is 2-colorable. Lovász [12] proved in 1976, that

$$
|\mathcal{E}| \leq\left(\begin{array}{c}
n \\
k-1
\end{array}\right)
$$

for a 3-color critical $k$-uniform hypergraph. Here we prove the following that can be considered as generalization of Lovász' result.

Theorem 1 Let $\mathcal{E} \subseteq\left(\begin{array}{c}{[m]} \\ k\end{array}\right)$ be a k-uniform set system on an underlying set $X$ of $m$ elements. Let us fix an ordering $E_{1}, E_{2}, \ldots E_{t}$ of $\mathcal{E}$ and a prescribed partition $A_{i} \cup B_{i}=E_{i}\left(A_{i} \cap B_{i}=\emptyset\right)$ for each member of $\mathcal{E}$. Assume that for all $i=1,2, \ldots, t$ there exists a partition $C_{i} \cup D_{i}=X\left(C_{i} \cap D_{i}=\emptyset\right)$, such that

\footnotetext{
${ }^{\dagger}$ Research is supported in part by NSERC

${ }^{\ddagger}$ Research is supported in part by NSERC

$\S$ Research is supported in part by Hungarian National Research Fund (OTKA) numbers T034702 and T037846

T Research is supported in part by Hungarian National Research Fund (OTKA) numbers T034702 and T037846 and by NSERC of the first author
}

1365-8050 @ 2005 Discrete Mathematics and Theoretical Computer Science (DMTCS), Nancy, France 
$E_{i} \cap C_{i}=A_{i}$ and $E_{i} \cap D_{i}=B_{i}$, but $E_{j} \cap C_{i} \neq A_{j}$ and $E_{j} \cap C_{i} \neq B_{j}$ for all $j<i$. (That is, the ith partition cuts the ith set as it is prescribed, but does not cut any earlier set properly.) Then

$$
t \leq\left(\begin{array}{c}
m \\
k-1
\end{array}\right)+\left(\begin{array}{c}
m \\
k-2
\end{array}\right)+\ldots+\left(\begin{array}{c}
m \\
0
\end{array}\right)
$$

Theorem 1 was motivated by the following sharpening of Sauer's bound for forbidden configurations. Let $F$ be a $k \times l$ 0-1 matrix, then forb $(m, F)$ denotes maximum $n$ such that there exists an $m \times n$ simple matrix $A$ such that no column and/or row permutation of $F$ is a submatrix of $A$. Furthermore, let $K_{k}$ denote the $k \times 2^{k}$ simple 0-1 matrix consisting of all possible columns.

Theorem 2 Let $F$ be contained in $F_{B}=\left[K_{k} \mid t \cdot\left(K_{k}-B\right)\right]$ for an $k \times(k+1)$ matrix $B$ consisting of one column of each possible column sum. Then forb $(m, F)=O\left(m^{k-1}\right)$.

We explain the the connection between Theorem 1 and Theorem 2.

The study of forbidden configurations is a problem in extremal set theory. The language we use here is matrix theory which conveniently encodes the problems. We define a simple matrix as a $(0,1)$-matrix with no repeated columns. Such a matrix can be thought of a set of subsets of $\{1,2, \ldots, m\}$ with the columns encoding the subsets and the rows indexing the elements. Assume we are give a $k \times l(0,1)$-matrix $F$. We say that a matrix $A$ has no configuration $F$ if no submatrix of $A$ is a row and column permutation of $F$ and so $F$ is referred to as a forbidden configuration (sometimes called trace). A variety of combinatorial objects can be defined by forbidden configurations. For a simple $m \times n$ matrix $A$ which is assumed to have no configuration $F$, we seek an upper bound on $n$ which will depend on $m, F$. We denote the best possible upper bound as forb $(m, F)$. Many results have been obtained about forb $(m, F)$ including [2],[3],[5].

At this point all values known for forb $(m, F)$ are of the form $\Theta\left(m^{e}\right)$ for some integer $e$. We completed the classification for $2 \times l$ matrices $F$ in [2] and for $3 \times l$ matrices $F$ in [6]. We also put forward a conjecture on what properties of $F$ drive the exponent $e$. Roughly speaking, we proposed a set of constructions and guessed that these constructions are sufficient to deduce the exponent $e$ in the expression $\Theta\left(m^{e}\right)$.

We use the notation $K_{k}$ to denote the $k \times 2^{k}$ simple matrix of all possible columns on $k$ rows. The basic result for forb $(m, F)$ is as follows.

Theorem 3 [Sauer [13], Perles and Shelah [14], Vapnik and Chervonenkis [15]] We have that forb $\left(m, K_{k}\right)$ is $\Theta\left(m^{k-1}\right)$.

In fact Theorem 3 is usually stated with forb $\left(m, K_{k}\right)=\left(\begin{array}{c}m \\ k-1\end{array}\right)+\left(\begin{array}{c}m \\ k-2\end{array}\right)+\cdots+\left(\begin{array}{c}m \\ 0\end{array}\right)$ but the asymptotic growth of $\Theta\left(m^{k-1}\right)$ was what interested Vapnik and Chervonenkis.

One easy observation is that if we let $A^{c}$ denote the 0-1-complement of $A$ then forb $\left(m, F^{c}\right)=\operatorname{forb}(m, F)$. Another observation is that if $F^{\prime}$ is a submatrix of $F$, then $\operatorname{forb}(m, F) \geq \operatorname{forb}\left(m, F^{\prime}\right)$. We let $K_{k}^{s}$ denote the $k \times\left(\begin{array}{l}k \\ s\end{array}\right)$ simple matrix of all possible columns of column sum $s$.

We use the notation $[A \mid B]$ to denote the matrix obtained from concatenating the two matrices $A$ and $B$. We use the notation $k \cdot A$ to denote the matrix $[A|A| \cdots \mid A]$ consisting of $k$ copies of $A$ concatenated together. We give precedence to the operation - (multiplication) over concatenation so that for example $[2 \cdot A \mid B]$ is the matrix consisting of the concatenation of $B$ with the concatenation of two copies of $A$.

According to an earlier unpublished result of Füredi [10] forb $(m, F)=O\left(m^{k}\right)$ for arbitrary $k \times l$ configuration $F$. The goal of this paper is to give sufficient conditions that ensure forb $(m, F)=O\left(m^{k-1}\right)$. 


\section{The boundary between $m^{k-1}$ and $m^{k}$}

Theorem 3 implies that simple configurations all have forb $(m, F)=O\left(m^{k-1}\right)$, thus we investigate $f$ 's with multiple columns. First, we show that which configurations $F$ have forb $(m, F)=\Omega\left(m^{k}\right)$ using the direct product construction. Let $A(k, 2)$ be defined as a minimal matrix with the property that any pair of rows has $\left[\begin{array}{l}1 \\ 1\end{array}\right]$ has both with 1's in some column and such that deleting a column of $A(k, 2)$ would violate this property.

Lemma 4 Let $F$ be a $k \times l$ configuration. forb $(m, F)=\Omega\left(m^{k}\right)$ if $F$ contains $2 \cdot K_{k}^{l}$ for $2 \leq l \leq k-2$ and $l=0, k$ or if $F$ contains $\left[2 \cdot K_{k}^{1} \mid A(k, 2)\right]$.

Proof: We find that $\operatorname{forb}(m, F)$ is $\Omega\left(m^{k}\right)$ if $F$ contains $2 \cdot K_{k}^{l}$ for $0 \leq l \leq k$ and $l \neq 1, k-1$. This

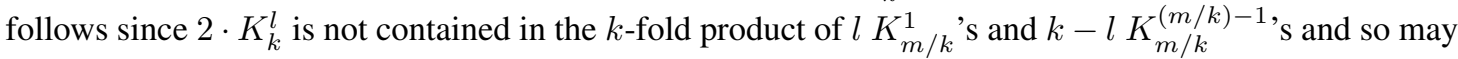
deduce forb $\left(m, 2 \cdot K_{k}^{l}\right)$ is $\Omega\left(m^{k}\right)$. To verify this for $2 \leq l \leq k-2$, we note that any pair of rows of $K_{k}^{l}$ has $\left[\begin{array}{ll}1 & 0 \\ 1 & 0\end{array}\right]$ and so if we have a submatrix that is a row and column permutation of $K_{k}^{l}$, we can only choose one row from either $K_{m / k}^{1}$ or from $K_{m / k}^{(m / k)-1}$. The verification for $K_{k}^{0}$ or $K_{k}^{k}$ is easier.

For $l=1$ (the case $l=k-1$ is the $(0,1)$-complement) we can no longer assert that any pair of rows of $K_{k}^{l}$ has $\left[\begin{array}{ll}1 & 0 \\ 1 & 0\end{array}\right]$ merely $\left[\begin{array}{l}0 \\ 0\end{array}\right]$ and so can choose two rows from the copy of $K_{m / k}^{1}$, one row from each of $k-2$ of the $K_{m / k}^{(m / k)-1}$ terms and generate a copy of $2 \cdot K_{k}^{1}$. (Theorem 5.1 of [6] shows that forb $\left(m, K_{k}^{1}\right.$ ) is $\left.\Theta\left(m_{k-1}\right)\right)$. This is fixed by considering a minimal matrix $A(k, 2)$ with the property that any pair of rows has $\left[\begin{array}{l}1 \\ 1\end{array}\right]$ has both with 1's in some column and such that deleting a column of $A(k, 2)$ would violate this. As above, we have that if $F$ contains $\left[2 \cdot K_{k}^{1} \mid A(k, 2)\right]$, then forb $(m, F)$ is $\Omega\left(m^{k}\right)$.

Lemma 4 leaves two possibilities if we want $\operatorname{forb}(m, f)$ be bounded away from $m^{k}$. Either $F$ is contained in a matrix $F_{B}=\left[K_{k} \mid t \cdot\left(K_{k}-B\right)\right]$ for an $k \times(k+1)$ matrix $B$ consisting of one column of each possible column sum or $F$ is contained in a matrix $\left[K_{k}^{0} \mid t \cdot C\right]$ where $C$ is a $k$-rowed simple matrix consisting of all columns which do not have 1's in both rows 1 and 2 and also with at least one 1. Note, that these are not mutually exclusive cases. Our main result Theorem 2 is that in the first case forb $(m, F)=O\left(m^{k-1}\right)$.

Proof of Theorem 2: Let $A$ be an $m \times n$ simple 0-1 matrix, and $B$ be a $k \times(k+1)$ matrix consisting of one column of each possible column sum. Suppose that $A$ does not have $F_{B}=\left[K_{k} \mid t \cdot\left(K_{k}-B\right)\right]$ as configuration. This implies that on a given $k$-tuple $L$ of rows either $K_{k}$ is missing, or if all possible columns of size $k$ occur on $L$, then $t \cdot\left(K_{k}-B\right)$ must be missing. This latter means, that for some $0 \leq s \leq k$, two columns of column sum $s$ occur at most $t-1$ times on $L$, respectively. Let $\mathcal{K}$ be the set of $k$-tuples of rows where the latter happens. Using Lemma 5 a set of columns of size $O\left(m^{k-1}\right)$ can be removed from $A$ to obtain $A^{\prime}$, so that for all $L \in \mathcal{K}$ a column (in fact two) is missing on $L$ in $A^{\prime}$. However, this implies that $K_{k}$ is not a configuration in $A^{\prime}$, thus by Theorem $3 A^{\prime}$ has at most $O\left(m^{k-1}\right)$ columns.

Let $\mathcal{K}$ be a system of $k$-tuples of rows such that $\forall K \in \mathcal{K}$ there are two $(k \times 1)$ columns, $\alpha_{K} \neq \beta_{K}$ specified. We say that a column $x$ of $A$ violates $\left(K, \alpha_{K}\right)$, if $\left.x\right|_{K}=\alpha_{K}$, similarly, $x$ violates $\left(K, \beta_{K}\right)$, if $\left.x\right|_{K}=\beta_{K}$. 
Lemma 5 Assume, that for every $K \in \mathcal{K}$ there are at most $t-1$ columns of $A$ that violate $\left(K, \alpha_{K}\right)$, and at most $t-1$ columns of $A$ violate $\left(K, \beta_{K}\right)$. Then there exists a subset $X$ of columns of $A$, such that $|X|=O\left(m^{k-1}\right)$ and no column of $A-X$ violates any of $\left(K, \alpha_{K}\right)$ or $\left(K, \beta_{K}\right)$.

Proof: It can be assumed without loss of generality that for all $K \in \mathcal{K} \alpha_{K}=\alpha$ and $\beta_{K}=\beta$ independent of $K$. Indeed, there are $2^{k} \times 2^{k}$ possible $\alpha_{K}, \beta_{K}$ pairs, that is a constant number of them .Thus, $\mathcal{K}$ can be partitioned into a constant number of parts, so that in each part $\alpha_{K}=\alpha$ and $\beta_{K}=\beta$ holds. We apply induction on $k$ using the simplification given above. $k=1$ is obvious.

Consider now $k \times 1$ columns $\alpha \neq \beta$. Assume first, that $\alpha \neq \bar{\beta}$. That is, there is a coordinate where $\alpha$ and $\beta$ agree, say both have 1 as their $\ell$ th coordinate. The case of a common 0 coordinate is similar. For the $i$ th row of $A$ we count how many columns have violation so that for some $K \in \mathcal{K}$ the $\ell$ th coordinate in $K$ is exactly row $i$. Let $\mathcal{K}_{i, \ell}$ be the set of these $k$-tuples from $\mathcal{K}$. Columns that have violation on $k$-tuples from $\mathcal{K}_{i, \ell}$ have 1 in the $i$ th row, let $A_{i, 1}$ denote matrix formed by the set of columns that have 1 in row $i$. If row $i$ is removed from $A_{i, 1}$, the remaining matrix $A_{i, 1}^{\prime}$ is still simple. Let $\mathcal{K}_{i, \ell}^{\prime}$ denote the set of $(k-1)$-tuples obtained from $k$-tuples of $\mathcal{K}_{i, \ell}$ by removing their $\ell$ th coordinate, $i$, furthermore let $\alpha^{\prime}\left(\beta^{\prime}\right.$, respectively) denote the $(k-1) \times 1$ column obtained from $\alpha(\beta)$ by removing the $\ell$ th coordinate, 1 . Note, that $\alpha^{\prime} \neq \beta^{\prime}$. A column of $A$ has a violation on $K \in \mathcal{K}_{i, \ell}$ iff its counterpart in $A_{i, 1}^{\prime}$ has a violation on the corresponding $K^{\prime} \in \mathcal{K}_{i, \ell}^{\prime}$. The number of those columns is at most $\mathrm{cm}^{k-2}$ by the inductive hypothesis. Since $\mathcal{K}=\cup_{i=1}^{m} \mathcal{K}_{i, \ell}$, we obtain that the number of columns of $A$ having violation on some $K \in \mathcal{K}$ is at most $m \cdot \mathrm{cm}^{k-2}$.

Let us assume now, that $\alpha=\bar{\beta}$. A subset $\mathcal{J} \subseteq \mathcal{K}$ is called independent if there exists an ordering $J_{1}, J_{2}, \ldots J_{g}$ of the elements of $\mathcal{J}$ such that for every $J_{i} \in \mathcal{J}$ there exists an $m \times 10$-1 column that violates $J_{i}$ and does not violate any $J_{j} \in \mathcal{J}$ for $j<i$. Let us call a maximal independent subset $\mathcal{B}$ of $\mathcal{K}$ a basis of $\mathcal{K}$. If a column of $A$ has a violation on $K \in \mathcal{K}$, then it has a violation on some $B \in \mathcal{B}$, as well. Indeed, either $K \in \mathcal{B}$ holds, or if $K \notin \mathcal{B}$, then by the maximality of $\mathcal{B}, K$ cannot be added to it as a $|\mathcal{B}|+1$ st element in the order, so the column having violation on $K$ must have a violation on $B \in \mathcal{B}$, for some $B$. By Theorem 1 for a basis $\mathcal{B}$ we have

$$
|\mathcal{B}| \leq\left(\begin{array}{c}
m \\
k-1
\end{array}\right)+\left(\begin{array}{c}
m \\
k-2
\end{array}\right)+\ldots+\left(\begin{array}{c}
m \\
0
\end{array}\right)
$$

since a column violating a $k$-tuple $B_{i}$ from $\mathcal{B}$, but none of $B_{j}$ for $j<i$, gives an appropriate partition of the set of rows. Thus, there could be at most $(2 t-2)\left[\left(\begin{array}{c}m \\ k-1\end{array}\right)+\left(\begin{array}{c}m \\ k-2\end{array}\right)+\ldots+\left(\begin{array}{c}m \\ 0\end{array}\right)\right]$ columns violating some $K \in \mathcal{K}$.

Proof of Theorem 1: We define a polynomial $p_{i}(\underline{x}) \in \mathbb{R}\left[x_{1}, x_{2}, \ldots, x_{m}\right]$ for each $E_{i}$ as follows.

$$
p_{i}\left(x_{1}, x_{2}, \ldots, x_{m}\right)=\prod_{a \in A_{i}}\left(1-x_{a}\right) \prod_{b \in B_{i}} x_{b}+(-1)^{k+1} \prod_{a \in A_{i}} x_{a} \prod_{b \in B_{i}}\left(1-x_{b}\right)
$$

Polynomials defined by (2) are multilinear of degree at most $k-1$, since the product $\prod_{e \in E_{i}} x_{e}$ cancels by the coefficient $(-1)^{k+1}$. Thus, they are from the space generated by monomials of type $\prod_{j=1}^{r} x_{i_{j}}$, for $r=0,1, \ldots k-1$. The dimension of this space over $\mathbb{R}$ is $\left(\begin{array}{c}m \\ k-1\end{array}\right)+\left(\begin{array}{c}m \\ k-2\end{array}\right)+\ldots+\left(\begin{array}{c}m \\ 0\end{array}\right)$. 
We shall prove that polynomials $p_{1}(\underline{x}), p_{2}(\underline{x}), \ldots p_{t}(\underline{x})$ are linearly independent over $\mathbb{R}$, which implies (1). Assume that

$$
\sum_{i=1}^{t} \lambda_{i} p_{i}(\underline{x})=0
$$

is a linear combination of the $p_{i}(\underline{x})$ 's that is the zero polynomial. Consider the partition $C_{t} \cup D_{t}=X$, and substitute $x_{c}=0$ if $c \in C_{t}$ and $x_{d}=1$ if $d \in D_{t}$ into (3). Then $p_{t}(\underline{x})=1$, but it is easy to see that $p_{k}(\underline{x})=0$ for $k<t$. This implies that $\lambda_{t}=0$. Now assume by induction on $j$, that $\lambda_{t}=\lambda_{t-1}=\ldots=\lambda_{t-j+1}=0$. Take the partition $C_{t-j} \cup D_{t-j}=X$ and substitute into (3) $x_{c}=0$ if $c \in C_{t-j}$ and $x_{d}=1$ if $d \in D_{t-j}$. Then, as before, $p_{t-j}(\underline{x})=1$, but $p_{k}(\underline{x})=0$ for $k<t-j$. This implies $\lambda_{t-j}=0$, as well. Thus, all coefficients in (3) must be 0 , hence the polynomials are linearly independent.

\section{References}

[1] R.P. AnsteE, Some Problems Concerning Forbidden Configurations, preprint.

[2] R.P. Anstee, J.R. Griggs, A. SAli, Small Forbidden Configurations, Graphs and Combinatorics 13(1997),97-118.

[3] R.P. Anstee, R. Ferguson, A. Sali, Small Forbidden Configurations II, Electronic J. Combin. 8(2001), R4 (25pp)

[4] R.P. Anstee, Z. FÜredI, Forbidden Submatrices, Discrete Math. 62(1986),225-243.

[5] R.P. Anstee, N. KAmoosi, Small Forbidden Configurations III, preprint.

[6] R.P. Anstee, A. SAli Small forbidden configurations IV: The 3-rowed case, Combinatorica, to appear.

[7] J. Balogh, B. Bollobás, Unavoidable Traces of Set Systems, to appear, Combinatorica.

[8] B. BollobÁs Extremal graph theory. London Mathematical Society Monographs, 11 Academic Press, Inc. [Harcourt Brace Jovanovich, Publishers], London-New York, 1978.

[9] P. Erdős, A.H. Stone, On the Structure of Linear Graphs, Bull. Amer. Math. Soc. 52(1946), 1089-1091.

[10] Z. FÜREDI, personal communication.

[11] T. KőVÁRI, V.T. Sós, P. TuRÁN On a problem of K. Zarankiewicz, Colloquium Math. 3 (1954) 50-57.

[12] L. LovÁsz Cromatic number of hypergraphs and linear algebra, Studia Sci. Math. Hung. 11 (1976) 113-114.

[13] N. Sauer, On the density of families of sets, J. Combin. Th. Ser A 13(1973), 145-147. 
[14] S. Shelah, A combinatorial problem: Stability and order for models and theories in infinitary languages, Pac. J. Math. 4(1972), 247-261.

[15] V.N. VAPNIK, A.YA. Chervonenkis, On the uniform convergence of relative frequencies of events to their probabilities, Th. Prob. and Applics. 16(1971), 264-280. 